\title{
Effects of adhesive characteristics of the catechol group on fiber-reinforced plastics
}

\author{
Polymer Journal (2011) 43, 944-947; doi:10.1038/pj.2011.91; published online 21 September 2011
}

\section{INTRODUCTION}

Mussels can strongly adhere to rocks by producing an amino acid (3,4-dihydroxyphenyl-L-alanine) called 'dopa', which contains the catechol group. ${ }^{1-5}$ The dopa-containing catechol group can strongly adhere to organic and/or inorganic surfaces, and its adhesion has been found to be reversible. Although there are several hypotheses regarding how this strong adhesion is achieved, either through hydrogen-bond interactions or covalent bonding between the catechol groups, the adhesion mechanism is not completely understood. ${ }^{6,7}$ However, these functions have fascinated many researchers regarding applications. For example, the research group of Messersmith and Miller, ${ }^{8-12}$ has mimicked the adhesion for application in adhesive sheets, and we have successfully synthesized mussel-mimetic high-performance adhesive resins. ${ }^{13}$ This finding indicates that the molecular interactions between catechols and inorganic surfaces can be used to develop macroscopic materials and has motivated us to utilize this catechol interaction to develop organic-inorganic hybrids such as glass-fiberreinforced plastics. Catechol derivatives are abundant in plant cell walls and in mussels. We previously reported the polymerization of the catechol-derivative caffeic acid (3,4-dihydroxycinnamic acid, DHCA, Figure 1a) in preparing wholly aromatic polyarylates, and their copolymerization with $p$-coumaric acid (4-hydroxycinnamic acid, 4HCA, Figure 1b) produced high-performance, biodegradable plant-derived plastics with high heat resistance above $150{ }^{\circ} \mathrm{C} .{ }^{14}$ Because DHCA has a role in the branching points, the copolymer was hyperbranched and had many catechol chain ends, as shown in Figure 1c. The mechanical strength of this copolymer resin in the highly oriented state is comparable to that of polycarbonates, though the strength in the non-oriented state is not as high (around
$30 \mathrm{MPa}$ ). If the resin-containing catechols are reinforced, thereby mimicking strong mussel adhesion, the mechanical weakness problem may be solved. Furthermore, the hyperbranched architecture with many catechol end groups may enhance and reinforce the mechanical strength.

In this study, we report the development of hybrid materials composed of poly (4HCA-coDHCA) with glass fibers or carbon fibers by changing the intramolecular interaction forces. In the case of the combination of poly(DHCA-co-4HCA) and fiber-coated sizing agents, the fracture strength of the materials is over $100 \mathrm{MPa}$, which is comparable to the strength of super engineering plastics derived from oil resources.

\section{EXPERIMENTAL PROCEDURE}

Synthesis of poly(DHCA-co-4HCA)

Caffeic acid ((4-hydroxycinnamic acid, DHCA; Tateyama Kasei, Toyama, Japan) and p-coumaric acid (4-hydroxycinnamic acid, 4HCA; Tateyama Kasei) were used as received, as monomers. The details of the synthesis of poly(DHCA-co-4HCA) are described elsewhere. ${ }^{13-15}$

\section{Preparation of fibers}

Glass fibers were obtained from Nippon Electric Glass (Shiga, Japan). The surfaces of the glass fibers obtained from the manufacturer were initially coated with a sizing agent composed of epoxy resin over a silane coupling agent (the details are unknown). Carbon fibers were obtained from Toray Industries (Tokyo, Japan), and the surfaces of these fibers were also initially coated with a sizing agent (the details are also unknown). To remove the sizing agent from these fibers, the fibers were washed with acetone and pure water several times. The epoxy resin and silane coupling agent could not be removed completely because they were assumed to form covalent bonds with the glass surface. After washing, they were treated with UV/ozone to introduce $\mathrm{OH}$ groups onto their surfaces. The introduction of the $\mathrm{OH}$ groups was evaluated indirectly by measuring the contact angles of water on the substrates, which were treated at the same time as shown in Table 2. After introducing the $\mathrm{OH}$ groups on their surfaces, the fibers were modified by the fluorine-modified silane coupling agent 3,3,3-trifluoropropyldimethoxy(methyl)silane to weaken the interaction between the filler fibers and the copolymers. We examined the fracture strengths of hybrids composed of these initial fibers, acetonewashed fibers, UV-ozone-treated fibers and fluorine-modified fibers to study how the surface interactions under the different surface conditions of these fibers affected the fracture strength of the hybrid composites.

\section{Molding conditions}

The hybrid samples used for the three-point bending test were prepared in a casting mold to a length of $50 \mathrm{~mm}$, width of $6.5 \mathrm{~mm}$ and thickness of $3.5 \mathrm{~mm}$. Poly (DHCA-co4HCA)s with various concentrations of filler fibers were set in a casting mold and pressed at $5 \mathrm{MPa}, 200{ }^{\circ} \mathrm{C}$ for $5 \mathrm{~min}$.

Three-point bending test

The three-point bending test was conducted by using a tensile materials testing machine (INSTRON, Canton City, MA, USA, 3365-L5). The samples were set over two fixtures $30 \mathrm{~mm}$ apart, and the center of each sample was pressed from the opposite direction. The fracture strength was defined as the maximum stress in the stress-strain curve.

\section{RESULTS AND DISCUSSION}

\section{Synthesis of poly(DHCA-co-4HCA)}

The chemical structures of the monomers are shown in Figure 1. Caffeic acid (4-hydroxycinnamic acid, DHCA; Tateyama Kasei) with 
a<smiles>O=C(O)/C=C/c1ccc(O)c(O)c1</smiles>

b<smiles>O=C(O)/C=C/c1ccc(O)cc1</smiles>

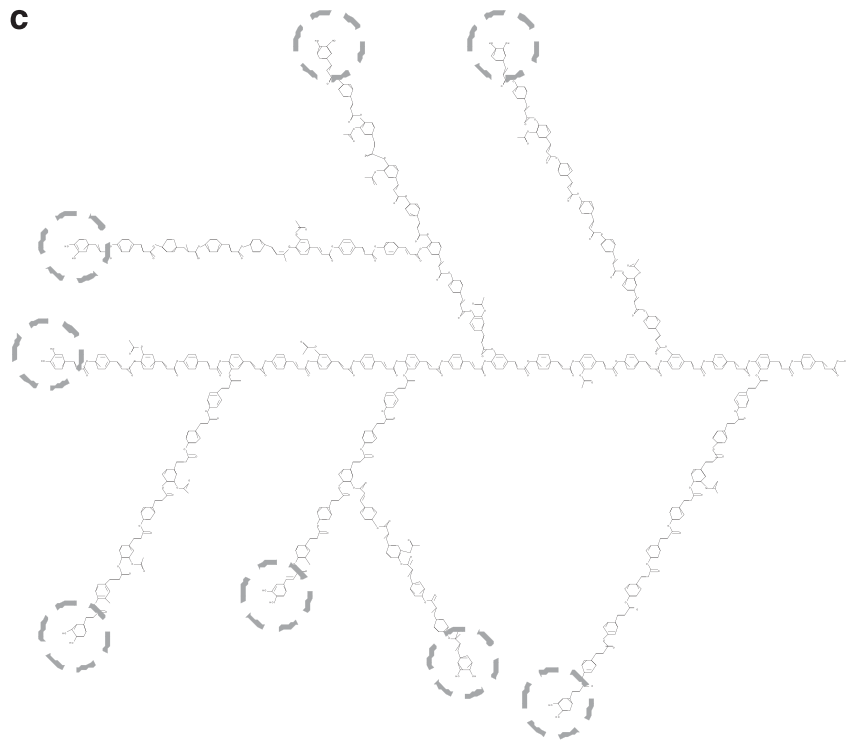

Figure 1 Chemical structure of (a) 3,4-dihydroxycinnamic acid (DHCA) and (b) 4-hydroxycinnamic acid (4HCA). (c) Chemical structure of poly(DHCA-co-4HCA) determined by its $\mathrm{H}^{1}-\mathrm{NMR}$, infrared and gel permeation chromatography spectra. The polymer chains were hyperbranched, and catechol groups were present at their chain ends (dotted circles). A full color version of this figure is available at Polymer Journal online.

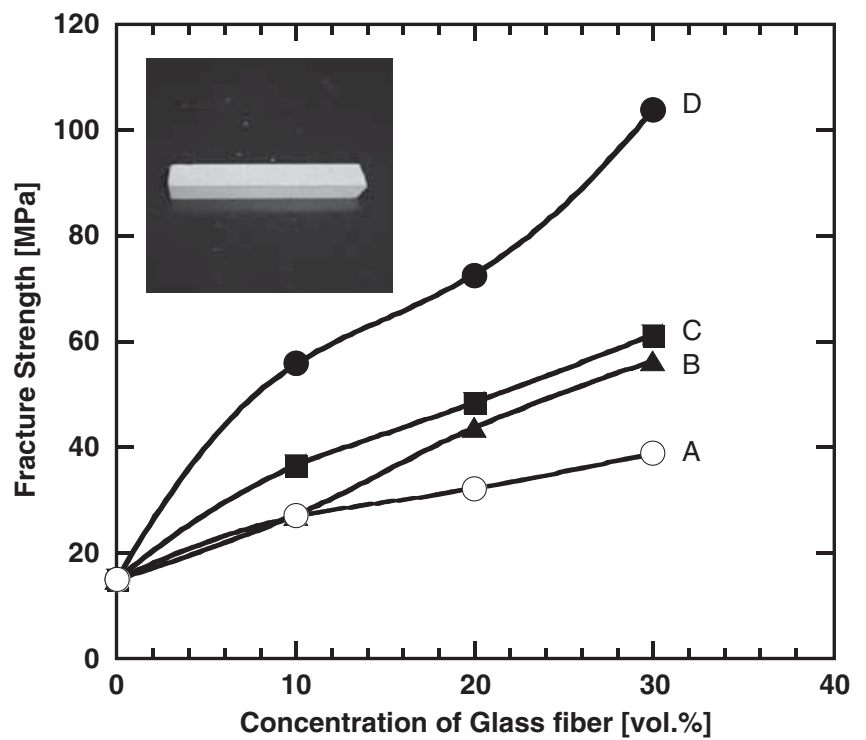

Figure 2 Fracture strength according to the three-point bending test as a function of the concentration of glass fibers. Open circles $(O)$ denote fluorinated glass fiber, filled triangles $(\boldsymbol{\Lambda})$ denote natural glass fiber, filled squares $(\boldsymbol{\square})$ denote ozone-treated glass fiber and filled circles $(\bullet)$ denote glass fiber with a sizing agent. The inset picture denotes the sample used for the three-point bending test $(3.5 \mathrm{~mm} \times 6.5 \mathrm{~mm} \times 50 \mathrm{~mm}$ ). A full color version of this figure is available at Polymer Journal online.

its catechol groups is shown in Figure 1a, and shows p-coumaric acid (4-hydroxycinnamic acid, 4HCA; Tateyama Kasei). The procedures regarding the copolymerization of
poly(DHCA-co-4HCA) are described elsewhere. ${ }^{13-15}$ The chemical structure of poly (DHCA-co-4HCA) is shown in Figure 1c. The high density of catechol groups at the chain ends can be expected to produce strong adhesive characteristics such as those shown by mussels.

\section{Preparation of fibers}

Glass and carbon fibers obtained from Nippon Electric Glass were covered by a sizing agent (the details are unknown) and were cut into $3 \mathrm{~mm}$ pieces. We named this initial fiber as condition D. The fiber samples were washed with acetone and pure water a few times to remove the sizing agent that covered them, and we named this bare and natural fiber as condition B. After removing the sizing agent, the samples were exposed to ozone gas for $20 \mathrm{~min}$ to introduce hydroxyl groups onto the fiber surfaces. We named this fiber as condition C. Each fiber was then fluorine-modified by 3,3,3-trifluoropropyldimethoxy(methyl)silane to weaken the interaction between the fibers and the matrix of the poly(DHCA-co-4HCA)s; we called this as condition A. Fiber A was used as the negative control. The details and modification procedures are further described in the experimental section.

\section{Three-point bending test}

To make rod-shaped samples for the threepoint bending test, fibers produced under various conditions were mixed with poly(DHCA-co-4HCA) in a mold. The sample shape was $3.5 \mathrm{~mm}$ in thickness, $6.5 \mathrm{~mm}$ in width and $50 \mathrm{~mm}$ in length, as shown in the inset picture of Figure 2. The samples were set on a stage supported by two points spaced $5 \mathrm{~cm}$ apart; the center of each sample was then pressed from the opposite direction. We obtained the stress-strain curve for each sample from a three-point bending test, and defined the fracture strength as the maximum value of the stress. Figure 2 shows the fracture strengths as a function of the concentration of the various conditions of the fibers. It was obvious that with increasing fiber concentration, the fracture strengths increased. Fluorinated fibers (case A) showed the lowest fracture strength at each fiber concentration, suggesting a weak interaction between the poly(DHCA-co-4HCA) matrix and fluorinated surfaces. In the case of bare glass fibers (case B), the fracture strength was superior to A because of the stronger interaction between the poly(DHCA-co-4HCA) matrix and the bare fibers. In the case of ozone-treated fibers (case C), the fracture strength increased slightly compared with that of the bare fibers (case B). The best performance was observed for samples featuring the sizing agent (case D). The fracture strength exceeded $100 \mathrm{MPa}$ for a sizing agent concentration of $30 \mathrm{vol} \%$, which 
Table 1 Fracture strength and progress rate of three-point bending test

\begin{tabular}{|c|c|c|c|c|c|}
\hline $\begin{array}{l}\text { Fracture strength of } \\
\text { various fiber conditions }\end{array}$ & $\begin{array}{l}\text { Original } \\
\text { (MPa) }\end{array}$ & $\begin{array}{c}\text { Fluorination (MPa) } \\
\text { (progression) }\end{array}$ & $\begin{array}{c}\text { Natural (MPa) } \\
\text { (progression) }\end{array}$ & $\begin{array}{l}\text { Ozone treatment } \\
\text { (MPa) (progression) }\end{array}$ & $\begin{array}{c}\text { Sizing agent } \\
\text { (MPa) (progression) }\end{array}$ \\
\hline PPS+glass fiber & 67 & $88(131 \%)$ & 101 (151\%) & $78(116 \%)$ & 115 (171\%) \\
\hline $\begin{array}{l}\text { Poly(DHCA-co-4HCA)+ } \\
\text { carbon fiber }\end{array}$ & 14 & $40(286 \%)$ & 55 (393\%) & 69 (493\%) & $110(786 \%)$ \\
\hline
\end{tabular}

Abbreviations: DHCA, 3,4-dihydroxycinnamic acid; 4HCA, 4-hydroxycinnamic acid; PPS, polyphenylenesulfide. Original indicates the sample without fibers.

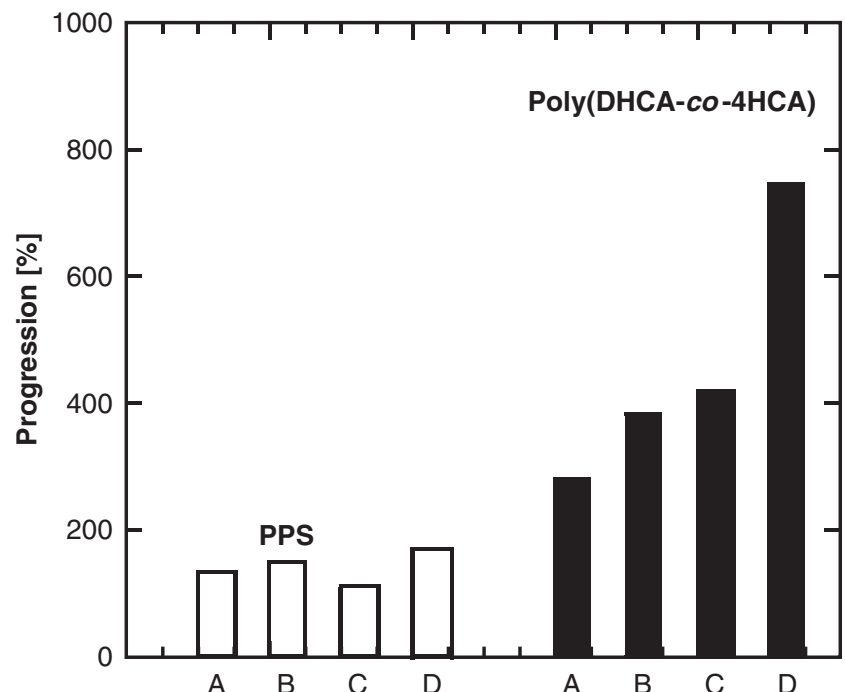

Figure 3 Progression of the fracture strength in samples containing glass fibers at $30 \mathrm{vol} \%$. The open bar and filled bar graphs denote polyphenylenesulfide (PPS) and poly(DHCA-co-4HCA), respectively. (A) Fluorinated glass fiber; (B) natural grass fiber; (C) ozone-treated glass fiber; and (D) glass fiber with the sizing agent (positive control).

Table 2 Water contact angle on the various fibers over substrates

\begin{tabular}{lccc}
\hline Glass condition & Natural (degree) & Ozone treatment (degree) & Fluorination (degree) \\
\hline Glass substrate & $44.5 \pm 3.1$ & $\sim 0$ & $67.7 \pm 0.5$ \\
Carbon substrate & $126 \pm 11.9$ & $28.7 \pm 4.6$ & $96.6 \pm 10.9$ \\
\hline
\end{tabular}

is equivalent to the strength of engineering plastics. These results suggest that the interaction force between the fiber surface and the matrix is one of the essential factors responsible for the fracture strength of hybrid materials. To clarify the effects of reinforcement by the catechol group, we evaluated another resin (polyphenylenesulfide (PPS)) without the catechol group under the same conditions using glass fibers (A-D). These results and the absolute values of the fracture strengths are summarized in Table 1.

We also measured the fracture strength of poly(DHCA-co-4HCA) with carbon fibers (surface conditions: A-D). In the case of carbon fibers containing $30 \mathrm{vol} \%$ sizing agent, the fracture strength also exceeded $100 \mathrm{MPa}$.

Figure 3 shows the progression of fracture strength with various glass fibers at $30 \mathrm{vol} \%$ from the fracture strength of the original polymer. For comparison, PPS samples under the same conditions are also shown. In the case of PPS with various conditions of glass fibers, the fracture strength could not exceed $200 \%$ in all the cases, and no correlations were observed with respect to the fiber surface conditions. In other words, the absolute values of the fracture strengths were higher than those of poly(DHCA-co-4HCA) as shown in Table 2, and the presence of the reinforced fibers had a limited effect because of the weak interactions between the fiber surface and the PPS matrix. In contrast, in the case of poly(DHCA-co-4HCA) with various conditions of glass fibers, the strength increased with increasing interactions between the fibers and the poly(DHCAco-4HCA)s. All cases exceeded a fracture strength of $200 \%$, and for the material containing the sizing agent (case D), it exceeded $700 \%$. These results indicate that the interaction between the fiber surface and the matrix is one of the most important factors responsible for fracture strength. In both fillers, the epoxy groups of the sizing agent were the most effective because of the strong covalent bond between the copolymer chain ends and the fillers. The fracture strengths of the materials in the copolymer resin were much higher than those in PPS, presumably because of the high reactivity of the catechol chain ends with the epoxy agents and low reactivity between PPS and the epoxyls. The strength of case C was $59-63 \%$ of that of case D. This value is surprisingly high because the strength of the hydrogen bonds is $20-30 \mathrm{pN}$, two orders of magnitude smaller than that of the covalent bonds $(2000-3000 \mathrm{pN})$. This phenomenon indicates that the interaction between the polymer matrix and the naked glass surface was very high, which may be attributed to the unknown interaction mechanism between catechols and the glass surface.

Figure 4 shows scanning electron microscope images of the fractured surface for cases A through D (A: fluorinated glass fiber; B: ozone-treated glass fiber; C: natural glass fiber; and D: glass fiber with sizing agent). In the case of PPS, it was confirmed that the surface of the glass fibers was not covered with PPS in all cases. In the case of poly (DHCA-co-4HCA), the case A glass fiber surface was similarly not covered by poly (DHCA-co-4HCA)s. In cases B, C and D, it was clearly confirmed that the surfaces of the glass fibers were covered by poly(DHCAco-4HCA)s. Furthermore, with an increase in the degree of interaction, the amount of poly(DHCA-co-4HCA) increased. These 

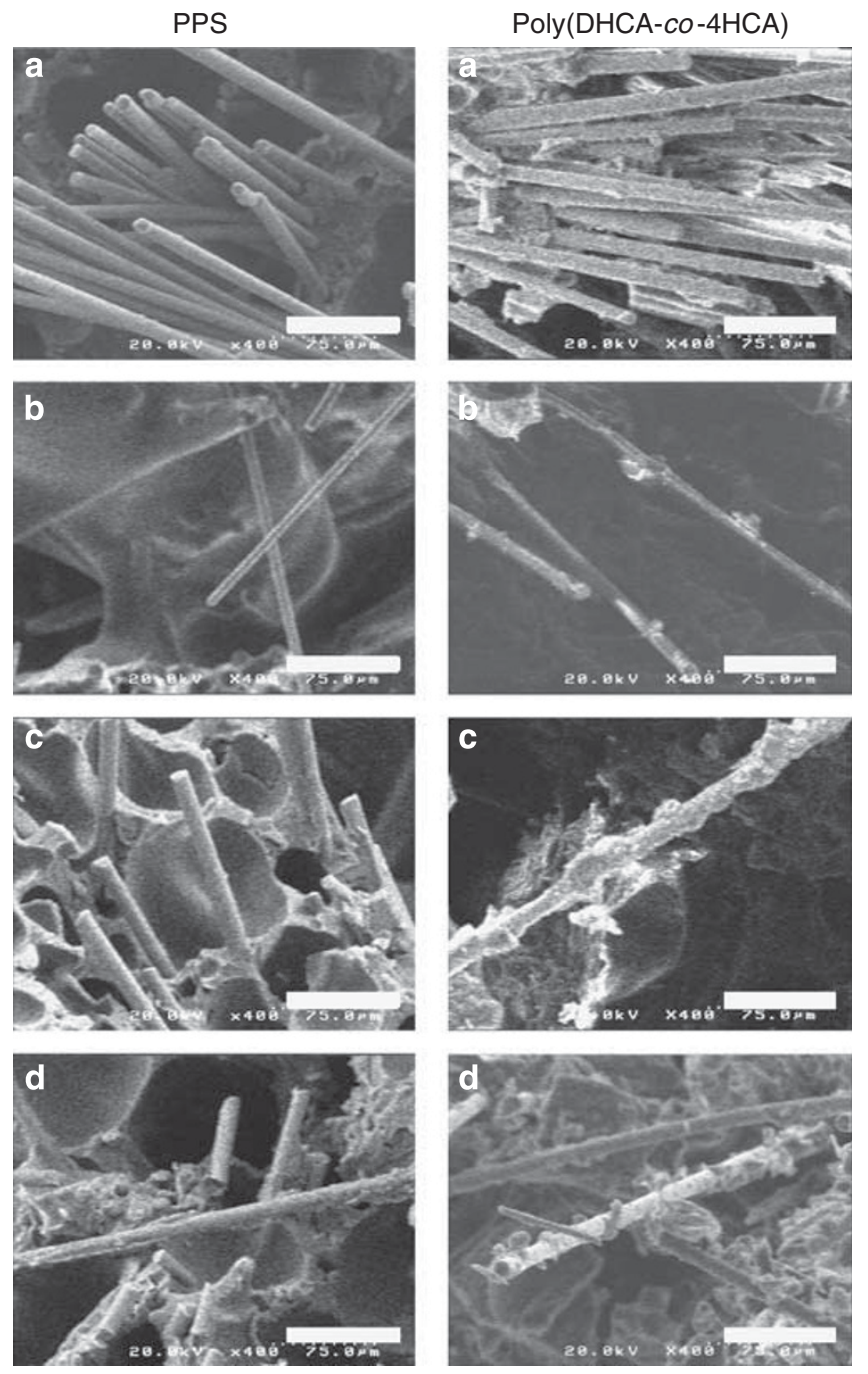

Figure 4 Scanning electron micrscopy image of fracture surface. (a) Fluorinated glass fiber, (b) ozonetreated glass fiber, (c) natural glass fiber, (d) glass fiber with sizing agent. Scale bars in the picture denote $50 \mu \mathrm{m}$.

results also support those shown in Figure 3, which suggest that the interaction between the fiber surface and the matrix is an important factor for fracture strength.

\section{SUMMARY}

We have synthesized a novel, plant-derived copolymer, poly(DHCA-co-4HCA), with catechol groups at its chain ends. Because the catechol group has a strong interaction with organic/inorganic surfaces, we investigated its effects on glass-fiber-reinforced plastics. Upon increasing the number of $\mathrm{OH}$ groups in the glass fibers, the fracture strengths also increased. When ozone-treated glass fibers were used, the mechanical strength was increased to over $400 \%$ of the original resin and approximately $60 \%$ of the covalently reinforced resins.

\section{CONFLICT OF INTEREST}

The authors declare no conflict of interest.

\section{ACKNOWLEDGEMENTS}

This research was partially supported by the Sasakawa Scientific Research Grant from the Japan Science Society, a Grant-in-Aid for
JST (Practical application research, Kaneko Project) and Iketani science and technology foundation. We sincerely offer our thanks to Professor Tadaoki Mitani for his valuable discussions.

Daisaku Kaneko, Keitaro Matsumoto, Shougo Kinugawa, Seiji Tateyama and Tatsuo Kaneko

School of Materials Science, Japan Advanced Institute of Science and Technology, Nomi City, Ishikawa, Japan E-mail: kaneko@jaist.ac.jp

1 Waite, J. H. \& Tanzer, M. L. Polyohenolic substance of Mytilus edulis: novel adhesive containing L-Dopa and hydroxyproline. Science 212, 1038-1040 (1981).

2 Lin, Q., Gourdon, D., Sun, C., Holten-Andersen, N., Anderson, T. H., Waite, J. H. \& Israelachvili, J. N. Adhesion mechanisms of the mussel foot proteins mfp-1 and mfp-3. Proc. Natl Acad. Sci. USA 104 (10), 3782-3786 (2007).

3 Weigemann, M. Adhesion in blue mussels (Mytilus edulis) and barnacles (genus Balanus): mechanisms and technical application. Aquat. Sci. 67, 166-176 (2005).

$4 \mathrm{Yu}, \mathrm{M}$. \& Deming, T. J. Synthetic polypeptide mimics of marine adhesives. Macromolecules 31, 4739-4745 (1988).

5 Yu, M., Hang, J. \& Deming, T. J. Role of L-3,4-dihydroxyphenylalanine in mussel adhesive proteins. J. Am. Chem. Soc. 121, 5825-5826 (1999).

6 Lee, T. J., Scerer, N. F. \& Messersmith, P. B. Singlemolecule mechanics of mussel adhesion. Proc. Natl Acad. Sci. USA 103, 12999-13033 (2006).

7 Waite, J. H. Mussel power. Nat. Mater. 7, 8-9 (2008).

8 Lee, H., Lee, B. P. \& Messersmith, P. B. A reversible wet/dry adhesive inspired by mussels and geckos. Nature 448, 338-341 (2007).

9 Gunawan, R. C., King, J. A., Lee, B. P., Messersmith, P. B. \& Miller, W. M. Surface presentation of bioactive ligands in a nonadhesive background using DOPA-tethered biotinylated poly(ethylene glycol). Langmuir 23, 10635-10643 (2007).

10 Lee, H., Dellatore, S. M., Miller, W. M. \& Messersmith, P. B. Mussel-inspired surface chemistry for multifunctional coatings. Science $\mathbf{3 1 8}, \mathbf{4 2 6}-\mathbf{4 3 0}$ (2007).

11 Lee, H., Lee, Y., Statz, A. R., Rho, J., Park, T. G. \& Messersmith, P. B. Substrate-independent layer-bylayer assembly by using mussel-adhesive-inspired polymers. Adv. Mater. 20, 1619-1623 (2008).

12 Lee, H., Rho, J. \& Messersmith, P. B. Facile conjugation of biomolecules onto surfaces via mussel adhesive protein inspired coatings. Adv. Mater. 21, 431-434 (2009).

13 Kaneko, D., Matsumoto, K., Kinugawa, K. \& Kaneko, T. Terminally-catecholized hyper-branched polymers with high performance adhesive characteristics. Plant Biotechnol. 27, 293-296 (2010).

14 Kaneko, T., Thi, T. H., Shi, D. J. \& Akashi, M. Environmentally degradable high-performance thermoplastics from phenolic phytomonomers. Nat. Mater. $\mathbf{5}$, 966-970 (2006).

15 Kaneko, D., Wang, S., Matsumoto, K., Kinugawa, S., Yasaki, K., Chi, D. H. \& Kaneko, T. Mussel-mimetic strong adhesive resin from bio-base polycoumarates. Polym. J. 43, 855-858 (2011). 